University of South Florida

DIGITAL COMMONS

Digital Commons @ University of

@ UNIVERSITY OF SOUTH FLORIDA

South Florida

1994

\title{
Modeling Rotating Flows with Neutral and Unstable Stratification
}

Samuel Hassid

Boris Galperin

University of South Florida, bgalperin@usf.edu

Follow this and additional works at: https://digitalcommons.usf.edu/msc_facpub

Part of the Life Sciences Commons

\section{Scholar Commons Citation}

Hassid, Samuel and Galperin, Boris, "Modeling Rotating Flows with Neutral and Unstable Stratification" (1994). Marine Science Faculty Publications. 1482.

https://digitalcommons.usf.edu/msc_facpub/1482

This Article is brought to you for free and open access by the College of Marine Science at Digital Commons @ University of South Florida. It has been accepted for inclusion in Marine Science Faculty Publications by an authorized administrator of Digital Commons @ University of South Florida. For more information, please contact digitalcommons@usf.edu. 


\title{
Modeling rotating flows with neutral and unstable stratification
}

\author{
Samuel Hassid \\ Environmental and Water Resources Engineering, Technion - Israel Institute of Technology, Haifa \\ Boris Galperin \\ Department of Marine Science, University of South Florida, St. Petersburg
}

\begin{abstract}
We investigate the effect of rotation on the structure of turbulence in neutral and convective mixed layers and assess the applicability of the Reynolds stress turbulence closure models to studies of deep convection. Unlike in the case of stable stratification, where rotational contribution is limited (Galperin et al., 1989), in neutral and convective flows this contribution is significant. The rotational terms endow eddy viscosity and eddy diffusivity with tensorial properties and algebraic complexity. In the present study we thoroughly investigate the mathematical features of this tensorial eddy viscosity-eddy diffusivity formulation. To ensure physical realizability of the model, we found it necessary to impose upper bounds on the dimensionless velocity and temperature gradients in the expressions for eddy coefficients. It was also found absolutely crucial to include in the model a limitation on the magnitude of the turbulence macroscale due to background rotation. This limitation is similar to the one imposed by stable stratification. The self-similar solutions derived from the model are compared with the experimental data by Fernando et al. (1991) and are shown to be in good agreement. Implications of the results for oceanographic modeling and for simulation of deep convection are discussed.
\end{abstract}

\section{Introduction}

Second moment or Reynolds stress turbulence closure models have been used to derive vertical mixing coefficients in models of ocean circulation. Although the oceanic turbulence is affected by numerous factors (density stratification, rotation, streamline curvature, etc.), only effects of stratification are usually explicitly accounted for in turbulence equations. Such a simplification can be justified on the basis of the length scale limitation due to stable stratification. Indeed, turbulence macroscale in stably stratified mixed layers cannot exceed the Ozmidov-Dougherty scale [Stillinger et al., 1983; Itsweire et al., 1993]. Galperin et al. [1989] (hereinafter referred to as paper 1) showed that this limitation is so powerful that it essentially confines the direct contribution of Coriolis terms to the total balance of turbulence correlations to within $10 \%$ of the nonrotational terms.

In neutral and convective mixed layers, however, the length scale limitation due to stable stratification does not apply, and one can expect that turbulent mixing is strongly affected by the planetary rotation (see paper 1). This is an important observation because turbu-

Copyright 1994 by the American Geophysical Union.

Paper number 94JC00149.

0148-0227/94/94JC-00149\$05.00 lence structure determines vertical mixing coefficients that enter Reynolds equations and have a strong effect on the dynamics and thermodynamics of the mean flow. Neutral and convective mixed layers are common in the ocean; they may develop in systems with strong evaporation (Mediterranean Sea), in midlatitude waters during seasonal cold weather outbreaks, and particularly in polar regions, where deep convection associated with the surface cooling and ice formation is one of the driving components of the deep ocean circulation [MEDOC Group, 1970]. The convection phenomenon is thus an integral part of the general ocean circulation; it feeds cold water into deep ocean currents, influences the thermohaline circulation and affects the global thermodynamic balance between the atmosphere and the ocean. It is clear that any realistic model of general ocean circulation must be capable of proper incorporation of the convection processes. There are ocean general circulation models (OGCM) available today that handle convective turbulence with various degrees of sophistication. For instance, Hakkinen et al. [1992] apply the Blumberg-Mellor circulation model [Blumberg and Mellor, 1983] to simulate deep convection in the Greenland Sea. To describe turbulent processes, their code incorporates the level $2 \frac{1}{2}$ model of Mellor and Yamada [1982]. This closure model, however, excludes the direct effect of rotation on turbulence correlations. It is not clear a priori whether or not such a simplification can be justified under convective conditions. Moreover, 
the results in paper 1 indicate that the opposite may be true. The purpose of the present study is therefore to further investigate this issue and to elucidate the importance of the explicit Coriolis terms in Reynolds stress and heat flux equations for realistic simulation of mean currents associated with the deep convection. It is important to note that the entire process of deep water formation is substantially three-dimensional (3-D) and should be handled by OGCMs. However, its deep convection phase can be approximately considered as quasi one-dimensional (1-D) in the vertical and can be thus described by a horizontally homogeneous turbulence closure model. Such an approach, which we pursue in the present study, is a deliberate simplification that allows us to concentrate on the physics of the vertical mixing in convective turbulence.

There are two more simplifications in our model. First, we consider only one stratifying factor, the temperature. In the real ocean, effects of salinity and compressibility (the so-called thermobaric instability) should also be taken into account. All these effects can be included in the present model if the equations are rewritten in terms of density using an appropriate equation of state (see, for instance, Mellor [1991]). Although feasible, consideration of all these factors would result in unnecessary complication of the present study, obscuring its main focus, the effect of rotation on convective flows, where it does not really matter what the source of convection is. Second, our model assumes that the flow is hydrostatic, while the oceanic deep convection is nonhydrostatic. Again, since this study is focused not on the real oceanic flows but rather on one aspect of their dynamics which can be elucidated even in hydrostatic simulations, we found it unnecessary to consider the nonhydrostatic effects at this point. All these simplifications are in line with the general methodology adopted by Mellor and Herring [1972] and Mellor [1973], who separate the process of modeling complex turbulent flows into two steps. First, the model is applied to simple and well-controlled laboratory data where basic model hypotheses are tested and constants are calibrated. Then, the model is used for simulations of complex flows, whereby the model's constants are kept invariant, but other terms in the equations, and thus other physical processes, are included. This paper deals with the first step in this process. Keeping all standard constants in the Mellor-Yamada closure model [Mellor and Yamada, 1982] invariant, we needed to add a new constant that appears in the rotational length scale limitation described in section 2 . The value of this constant, set by the energy balance considerations, was tested against experimental data of Fernando et al. [1991]. Although the resulting model gives a simplified, quasi 1-D description of convective turbulence, it nevertheless provides important implications for general 3-D modeling of convective flows.

This paper essentially extends the analysis of paper 1 to neutral and unstably stratified flows. Section 2 provides the mathematical formulation of the problem. In the cases under consideration the Coriolis- related terms are of the same order of magnitude as other terms in the expressions for eddy viscosity-eddy diffusivity, and small parameter expansions derived in paper 1 are inapplicable. Moreover, certain parameter ranges of Coriolis, stratification, and mean shear terms render these expressions physically unrealizable, which manifest in negative or infinite values of the mixing coefficients, unrealistic distributions of turbulence energy, etc. The appropriate realizability conditions that prevent these problems are described in section 3 . Similar realizability conditions that limited the range of variation of the stratification parameter in the expressions for eddy viscosity-diffusivity were derived by Galperin et al. [1988] (hereinafter referred to as paper 2), who excluded rotational terms from the turbulence equations. In the present study, given the complexity of the Reynolds stress and heat flux equations, in which both components of the Coriolis vector are retained the realizability conditions become far more complicated. Section 4 demonstrates the complexity and the wide range of variation of the turbulence exchange coefficients in neutral and unstably stratified flows with rotation. Section 5 compares model predictions with some available experimental data and demonstrates that an agreement between the model and the data is only possible when the turbulence macroscale limitation due to rotation is included in the model. Section 6 discusses the results and emphasizes implications for modeling of deep convection.

\section{Mathematical Formulation}

The present study extends the previous works by $\mathrm{Mel}$ lor and Yamada [1982], Kanthe et al. [1989], and in papers 1 and 2, in that it considers the direct effect of Coriolis terms on turbulence correlations in neutral and unstably stratified boundary layers with background rotation. The study is based upon the $2 \frac{1}{2}$ level model of Mellor and Yamada [1982] modified in paper 2. The turbulence part of the model consists of the algebraic Reynolds stress and heat flux equations,

$$
\begin{aligned}
\overline{u_{i} u_{j}} & =\frac{\delta_{i j}}{3} q^{2}-\frac{3 l_{1}}{q}\left[\overline{u_{k} u_{i}} \frac{\partial U_{j}}{\partial x_{k}}+\overline{u_{k} u_{j}} \frac{\partial U_{i}}{\partial x_{k}}\right. \\
& -C_{1} q^{2}\left(\frac{\partial U_{j}}{\partial x_{i}}+\frac{\partial U_{i}}{\partial x_{j}}\right)+\left(g_{i} \overline{u_{j} \theta}+g_{j} \overline{u_{i} \theta}\right) \\
& \left.+f_{k}\left(\epsilon_{i k l} \overline{\overline{u_{l} u_{j}}}+\epsilon_{j k l} \overline{u_{l} u_{i}}\right)+\frac{2 q^{3}}{3 \Lambda_{1}} \delta_{i j}\right]
\end{aligned}
$$

and

$\overline{u_{i} \theta}=-\frac{3 l_{2}}{q}\left[\overline{u_{i} u_{k}} \frac{\partial \Theta}{\partial x_{k}}+\overline{\theta u_{k}} \frac{\partial U_{i}}{\partial x_{k}}+\beta g_{i} \overline{\theta^{2}}+f_{k} \epsilon_{i k l} \overline{u_{l} \theta}\right]$

where $U_{i}$ and $u_{i}$ are the mean and fluctuating velocities, $\Theta$ and $\theta$ are the mean and fluctuating potential temperature, $g_{i}$ is the acceleration due to gravity, $\beta$ is the volumetric expansion coefficient, $q^{2} \equiv \overline{u_{k}^{2}}$, and $f_{k}=$ $2 \Omega_{k}=\left(0, f_{y}, f\right)$ is the Coriolis vector, $\Omega=\left(\sum_{k} \Omega_{k}^{2}\right)^{1 / 2}$ 
being the angular velocity of Earth's rotation. These equations are solved in conjunction with the turbulence energy and temperature variance equations

$$
\frac{D q^{2}}{D t}-\frac{\partial}{\partial x_{k}}\left(q l S_{q} \frac{\partial q^{2}}{\partial x_{k}}\right)=-2 \overline{u_{k} u_{l}} \frac{\partial U_{k}}{\partial x_{l}}-2 \beta g_{k} \overline{u_{k} \theta}-2 \frac{q^{3}}{\Lambda_{1}},
$$

$$
\overline{\theta^{2}}=-\frac{\Lambda_{2}}{q} \overline{u_{k} \theta} \frac{\partial \Theta}{\partial x_{k}} .
$$

Here, $S_{q}$ is the nondimensional vertical exchange coefficient for the kinetic energy, and the various length scales of turbulence are related to the master length scale $l$ following Mellor and Yamada [1982]:

$$
\left(l_{1}, l_{2}, \Lambda_{1}, \Lambda_{2}\right)=\left(A_{1}, A_{2}, B_{1}, B_{2}\right) l,
$$

where

$$
\left(A_{1}, A_{2}, B_{1}, B_{2}\right)=(0.92,0.74,16.6,10.1) .
$$

The additional constant $C_{1}$ appearing in (1) is related to other model's constants (see paper 1) and is equal to 0.08 . The length scale can either be prescribed or calculated using an additional transport equation for $q^{2} l$ [see Mellor and Yamada, 1982]. For flows near rigid walls the length scale consistent with the above formulation is equal to $\kappa z$; $z$ being the distance from the nearest surface and $\kappa$ being the von Kármán constant $(\kappa=0.4)$. Equations (1)-(4) are solved in the boundary layer approximation, where only vertical gradients are retained.

Similarly to paper 1 , let us define the following dimensionless variables:

$$
\begin{gathered}
R u \equiv \frac{l}{q} \frac{\partial U}{\partial z} \\
R v \equiv \frac{l}{q} \frac{\partial V}{\partial z}, \\
R o_{y}^{-1} \equiv \frac{l}{q} f_{y} \\
R o_{z}^{-1} \equiv \frac{l}{q} f \\
G_{H} \equiv-\left(\frac{l}{q}\right)^{2} \beta g \frac{\partial \Theta}{\partial z} .
\end{gathered}
$$

Equations (1)-(4) admit tensorial eddy viscosityeddy diffusivity representation relating the Reynolds stress components $\overline{u w}, \overline{v w}$, and heat flux $\overline{w \theta}$ to the mean velocity and temperature gradients, $\partial U / \partial z, \partial V / \partial z$, and $\partial \Theta / \partial z$, respectively. In the nondimensional form these relations are

$$
\begin{aligned}
-\frac{\overline{u w}}{q^{2}} & =S_{u u} R u+S_{u v} R v+S_{u \theta} G_{H}, \\
-\frac{\overline{v w}}{q^{2}} & =-S_{v u} R u+S_{v v} R v-S_{v \theta} G_{H}, \\
-\frac{l}{q^{3}} \beta g \overline{w \theta} & =-S_{\theta \theta} G_{H} .
\end{aligned}
$$

Algebraic equations relating $S_{u u}, S_{u v}, S_{u \theta}, S_{v u}, S_{v v}$, $S_{v \theta}$, and $S_{\theta \theta}$ to $G_{H}, R u, R v, R o_{z}^{-1}$, and $R o_{y}^{-1}$ are quite complex; they were obtained using a symbolic manipulation language (Mathematica in this study, REDUCE in paper 1). (These equations are available from the authors.) Significant simplifications of these expressions are only possible in a few special cases, such as $G_{H}=0$ (neutral stratification), when $S_{u \theta}$ and $S_{v \theta}$ become irrelevant; $R o_{z}^{-1}=0$, when $S_{u v}, S_{v u}$, and $S_{v \theta}$ vanish; and $R o_{y}^{-1}=0$, when $S_{u \theta}$ and $S_{v \theta}$ vanish. In the case of neutral stratification the dynamic coupling between the velocity and temperature fields disappears, and the third equation in (9) becomes an eddy diffusivity formulation for a passive scalar, $-\overline{w \theta}=q l S_{\theta \theta} \partial \Theta / \partial z$. Note that in the general case, $S_{u v} \neq S_{v u}$ (although the values of these quantities are very close, especially when they are significant); $S_{u u}, S_{v v}, S_{\theta \theta}, S_{u v} R o_{z}$, and $S_{v u} R o_{z}$ depend on $R u R o_{y}^{-1}$ and $R v R o_{z}^{-1} R o_{y}^{-1}$ rather than on $R u$ and $R v$. For purely convective turbulence with no shear $(R u=0$ and $R v=0), S_{u u}, S_{v v}, S_{\theta \theta}, S_{u v} R o_{z}, S_{v u} R o_{z}, S_{u \theta} R o_{y}$ and $S_{v \theta} R o_{y} R o_{z}$ are all even functions of $R o_{y}$ and $R o_{z}$. Furthermore, in case $R o_{y}^{-1}=0, S_{\theta \theta}$ becomes independent of $\mathrm{Ro}_{2}^{-1}$. Of course, the model is identical with the modified $2 \frac{1}{2}$ model when the components of rotation are set to zero. Note that the signs in (9) are chosen in such a way that all exchange coefficients are positive for positive $\mathrm{Ro}_{y}^{-1}$ and $\mathrm{Ro}_{\mathrm{z}}^{-1}$.

Galperin and Kantha [1989] applied this model to neutral boundary layers with axial $\left(R o_{y}^{-1}=0, R o_{z}^{-1} \neq\right.$ $0)$ and spanwise $\left(R o_{y}^{-1} \neq 0, R o_{z}^{-1}=0\right)$ rotation, while Galperin and Mellor [1991] considered the combined effect of spanwise rotation and streamline curvature. Model predictions in both studies agree well with other modeling efforts and experimental data available in mechanical engineering. Galperin and Kantha [1989] showed that strong rotation tends to suppress turbulence in either axial or spanwise configuration. In the case of axial rotation, Coriolis terms cause monotonic decrease of the eddy viscosity until it falls to zero, indicating the extinction of 3-D turbulence. In the case of spanwise rotation the picture is more subtle. Rotational terms cause redistribution of turbulence energy between $\overline{u^{2}}$ and $\overline{w^{2}}$ in such a way that for moderate rotation rates the flow may be stabilized or destabilized. However, an increase in the destabilizing rotation rate leads to flow restabilization which culminates in the extinction of 3-D turbulence. Similar analysis was performed by Tritton [1992], who also considered stabilization, destabilization, and restabilization of shear layers by spanwise rotation and derived criteria for these phenomena analogous to those by Galperin and Kantha [1989].

\section{Realizability Conditions}

Paper 2 describes the constraints imposed on the value of $G_{H}$ for both stable and unstable stratification. On the stable side the constraint stems from the length scale limitation first used by André et al. [1978],

$$
l N / q \leq 0.53
$$


where $N \equiv(-\beta g \partial \Theta / \partial z)^{1 / 2}$ is the Brunt-Väisälä frequency. The nature of $(10)$ has been extensively discussed in paper 1 ; essentially, it constitutes that to overturn, the kinetic energy of an eddy, $q^{2} / 2$, should exceed the counteracting potential energy of the background stable stratification $(\mathrm{Nl})^{2} / 2$. On the unstable side the constraint on the value of $G_{H}$ is dictated by the requirement that the buoyant production does not exceed the dissipation; it leads to

$$
G_{H} \leq \mathbf{0 . 0 2 3 3} \text {. }
$$

Inequalities (10) and (11) effectively limit the values of $S_{u v}=S_{v v}$ and $S_{\theta \theta}$, which are the only nontrivial, nondimensional vertical mixing coefficients when $R o_{y}^{-1}=R o_{z}^{-1}=0$.

One would expect that when $R o_{y}^{-1}, R o_{z}^{-1} \neq 0$, similar constraints are necessary to ensure physical realizability of the eddy viscosity-eddy diffusivity formulation given by (9). This issue was not addressed in the previous papers and will be dealt with in sections 3.1 and 3.2. Needless to say, the new constraints will be more complex with the presence of rotational terms, given that stabilizing rotation can be combined with destabilizing stratification and also that it might be necessary to restrict the range of variation of $R u$ and $R v$, something that was not necessary in the absence of rotation.

\subsection{The Length Scale Limitations Due to Rota- tion}

When a fluid element with the kinetic energy $q^{2} / 2$ travels in the presence of rotation in the radial direction, some of its energy, as a result of the action of the kinematic centrifugal force $\Omega^{2} r$, is transformed into potential energy (here $\Omega$ and $r$ are the angular velocity of rotation and the distance from the axis of rotation, respectively). Rationale similar to that used to derive (10) leads to a limitation on the length scale due to rotation. It constitutes that an overturn of an eddy requires that its kinetic energy exceeds the potential energy $(\Omega l)^{2} / 2$ associated with the centrifugal force and leads to inequality

$$
l \Omega / q \leq c,
$$

where $c$ is an empirical constant that needs to be determined. To conform with this energy consideration, the value of the constant $c$ has been set to 1 ; as will be shown in section $5,(12)$ with $c=1$ is supported by the experimental results of Boubnov and Golitsyn [1990] and Fernando et al. [1991], in which $R o_{y}^{-1}=0$.

Inequality (12) is equivalent to

$$
\left(R o_{y}^{-2}+R o_{z}^{-2}\right)^{1 / 2} \leq 2
$$

from which two other constraints can be inferred

$$
\begin{aligned}
& \left|R o_{y}^{-1}\right| \leq 2 \\
& \left|R o_{z}^{-1}\right| \leq 2
\end{aligned}
$$

These inequalities can be used for general analysis of the effect of the Coriolis-related terms on the Reynolds stress and heat flux equations. Recall that in paper 1 , equation (39), this effect was given by the matrix equation

$$
\mathbf{X}=\left(\mathrm{I}+R o_{y}^{-1} \mathbf{V A}_{y}+R o_{z}^{-1} \mathbf{V A}_{z}\right)^{-1} \mathbf{V B},
$$

where $\mathbf{X}$ is the matrix column of Reynolds stresses and turbulent heat fluxes, $I$ is the unit matrix, and the matrices $\mathbf{V}, \mathbf{B}, \mathbf{A}_{y}$, and $\mathbf{A}_{\mathbf{z}}$ were defined in paper 1 . Since Coriolis terms enter (14) linearly in the expression within the brackets, their relative contribution can be estimated from the inequality

$$
\begin{aligned}
\| \mathrm{I} & +R o_{y}^{-1} \mathbf{V A}_{y}+R o_{z}^{-1} \mathbf{V A}_{z} \| \leq 1 \\
& +\left|R o_{y}^{-1}\right|\left\|\mathbf{V A}_{y}\right\|+\left|R o_{z}^{-1}\right|\left\|\mathbf{V A}_{z}\right\|,
\end{aligned}
$$

where $\|\cdot\|$ denotes the matrix norm. In paper 1 , appendix B, it was shown that for neutral and convective flows, $\left\|\mathbf{V A}_{\mathbf{z}}\right\|$ generally does not exceed 20, while $\left\|V_{y}\right\|$ may become as large as 100 . Combined with (13b) and (13c), (15) thus indicates that the relative effect of the Coriolis terms on turbulence structure may be quite significant. Even for small $R o_{y}^{-1}$ and $\left|R o_{z}^{-1}\right|$, say, of the order of $10^{-1}$, the Coriolis terms may exceed all other contributions by an order of magnitude.

For illustration, let us consider parameter values typical of oceanic turbulence. For $q \sim 10^{-2} \mathrm{~m} \mathrm{~s}^{-1}$ and $\left|R o_{z}^{-1}\right|, R o_{y}^{-1} \sim 10^{-4} \mathrm{~s}^{-1},(12)$ gives $l \leq 100 \mathrm{~m}$. Assuming that the turbulence macroscale is within $1 / 10$ of the mixed layer depth, one concludes that the lengthscale limitation is mainly felt in the deepest regions of convective systems, such as the "chimneys" in the polar oceans. On the other hand, even relatively shallow convective layers, such as those that occur as a result of the seasonal cooling and have inverse turbulent Rossby numbers of the order of $10^{-1}$, may still be strongly affected by Coriolis terms, particularly those related to $R o_{y}^{-1}$.

Clearly, effects of rotation on neutral and convective turbulence cannot be neglected in numerical models. The proper incorporation of these effects in the present model requires that (12) be included as an external constraint. The application of this constraint is straightforward in flows where rotation has only one component. If $\Omega$ has more than one component, such as $\Omega_{y}$ and $\Omega$ in the geophysical context, the use of (12) becomes more complex. For instance, one could think of the constraint on $l$ based upon (12), but employing the largest of $\Omega_{y}$ and $\Omega$. In the present study, (13a) has been chosen as the length scale limitation because it is invariant under transformations of the coordinate system.

It is important to mention that the length scale limitation is not a part of conventional Reynolds stress closure models and thus it should be imposed as an external condition. It pertains to spectral dynamics of turbulence; wave interaction and turbulence twodimensionalization, the processes usually excluded in Reynolds average-type models. There exist more so- 
phisticated, spectral models of turbulence with rotation (see, for instance, Cambon and Jacquin [1989] and Jacquin et al. [1990]), but they are yet to be adapted for practical applications in finite difference Reynolds average models.

3.2. Limitations on $G_{H}, R u$ and $R v$ for Neutral and Convective Turbulence

The nondimensional mixing coefficients given by (9) explicitly depend on $R u, R v, G_{H}, R o_{y}^{-1}$, and $R o_{z}^{-1}$. When these coefficients are straightforwardly used in numerical simulations, they can lead to unrealistic results or singularities. The source of the problem is the existence of nonphysical solutions to the fully algebraic set of equations for turbulence correlations in the local equilibrium, or level 2 model. A similar problem occurs in the original $2 \frac{1}{2}$-level model of Mellor and $\mathrm{Ya}$ mada [1982], where the explicit dependence of the mixing coefficients on $(\partial U / \partial z)^{2}+(\partial V / \partial z)^{2}$ leads to the existence of a parameter range that renders the system physically unrealizable. The problem with the original $2 \frac{1}{5}$-level model was resolved in paper 2 by elimination of the $(\partial U / \partial z)^{2}+(\partial V / \partial z)^{2}$ term from the expressions for the mixing coefficients. In the present case, however, such a simple remedy is not possible, and one needs to introduce some rules which should limit the range of variation of $R u, R v, G_{H}, R o_{y}^{-1}$, and $R o_{z}^{-1}$ in the expressions for the mixing coefficients. The choice of these rules is not unique, and thus the limiting manifold is not well defined. However, it is assumed that the results of simulations will not be overly sensitive to the particular choice of limitations as long as they don't disturb the mean field and preserve turbulence correlations as physically realizable.

The range of variation of $R o_{y}^{-1}$ and $R_{z}^{-1}$ is limited according to (13b) and (13c). To restrict the variation of $R u, R v$, and $G_{H}$, one needs three additional constraints. One of them is related to the distribution of turbulence energy among its components. In paper 2 it was shown that with rotational terms excluded, $\overline{u^{2}} / q^{2}+\overline{v^{2}} / q^{2}+\overline{w^{2}} / q^{2}=1+O\left(a^{2}\right)$, where $a$ is a small parameter characterizing the deviation from the local isotropy. For the local equilibrium or the level 2 model it is easy to see that the sum $\overline{u^{2}} / q^{2}+\overline{v^{2}} / q^{2}+\overline{w^{2}} / q^{2}$ is equal to 1 identically, with or without rotation. With Coriolis terms included, when $\overline{u^{2}} / q^{2}, \overline{v^{2}} / q^{2}$ and $\overline{w^{2}} / q^{2}$ are expressed in terms of the mixing coefficients, then parameter ranges are found in which their sum exceeds the value of 1 . Excluding these ranges and thus demanding that

$$
\overline{u^{2}} / q^{2}+\overline{v^{2}} / q^{2}+\overline{w^{2}} / q^{2} \leq 1,
$$

one derives the limiting values $G_{H_{\max }}, R u_{\max }$ and $R v_{\max }$ or the realizability conditions, for which the matrix of the mixing coefficients in (9) remains physically meaningful. In actual simulations the values of $R u, R v$, and $G_{H}$ will not be allowed to exceed $G_{H_{\max }}, R u_{\max }$, and $R v_{\max }$ in calculations of the exchange coefficients.
Mean fields and gradients in the rest of the model, of course, will not be changed.

With rotational terms retained, (16) results in a constraint which is quartic in $R u$ and $R v$ and cubic in $G_{H}$

$$
\Phi\left(R o_{y}^{-1}, R o_{z}^{-1}, R u, R v, G_{H}\right) \leq 0 .
$$

The exact form of the function $\Phi$ is algebraically complicated and is not given here. Two additional constraints are:

$$
\begin{gathered}
R u_{\max } / R v_{\max }=R u / R v \\
\frac{\left(R u_{\max }^{2}+R v_{\max }^{2}\right)^{1 / 2}}{G_{H_{\max }}}=\frac{\left(R u^{2}+R v^{2}\right)^{1 / 2}}{G_{H}}
\end{gathered}
$$

Equation (18) is equivalent to limiting the length of the nondimensional velocity shear vector without affecting its direction. In (19) the gradient Richardson-numberlike parameter based on $R u, R v$, and $G_{H}$ is kept constant.

It is clear that (16)-(19) may produce numerous combinations of the limiting parameters $R u_{\max }, R v_{\max }$, and

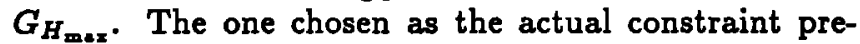
serves the signs of $R u$ and $R v$ and uses parameters with the smallest absolute values.

The dependence of $G_{H_{\max }}$ on $R o_{y}^{-1}$ and $R o_{z}^{-1}$ for purely convective turbulence is shown in Figure 1 . Here, as well as in the rest of the figures to follow, the ranges of $R o_{y}^{-1}$ and $R o_{z}^{-1}$, for generality, are set between -2 and 2, although in geophysical applications, $R o_{y}^{-1}$ is always positive. However, in some cases negative $\mathrm{Ro}_{y}^{-1}$ is equivalent to negative $R u$, such that the present range of Rossby numbers allows one to account for different directions of the mean flow. One can see that $G_{H_{\max }}$ possesses a plane symmetry with respect to the $R o_{y}^{-1}=0$ axis. For $R o_{y}^{-1}=R o_{z}^{-1}=0$ the maximum value of $G_{H}$ is $\mathbf{0 . 0 2 3 3}$, consistent with paper 2. For $R o_{y}^{-1}=0$ the value of $G_{H_{\operatorname{mox}}}$ is independent of $R o_{z}^{-1} . G_{H_{\max }}$ increases with increasing $\left|R o_{y}^{-1}\right|$, having a particularly sharp rise in the vicinity of $R o_{x}^{-1}=0$, where $G_{H_{\max }}$ can increase by more than an order of magnitude compared to the case $R o_{y}^{-1}=0$.

A nonzero shear renders the situation more complicated, even in the case of neutrally stratified media, because $R u_{\max }$ and $R v_{\max }$ depend on the direction of the velocity vector. For convective turbulence with shear (Figure 2) it can be seen that $R u_{\max }$ and $R v_{\max }$ have their minima in the vicinity of $R o_{y}^{-1}=R o_{z}^{-1}=0$ and generally grow with $R o_{z}^{-1}$ and $R o_{y}^{-1}$, although there are certain directions where they decrease. For the case of neutral stratification the dependencies are quite similar and are not shown.

The use of the constraints or realizability conditions ensures that components of the matrix of exchange coefficients are well behaved and do not attain infinite or negative values, and that the discriminant of the matrix is positively definite. These realizability conditions should be incorporated into all numerical simulations employing the present model. 


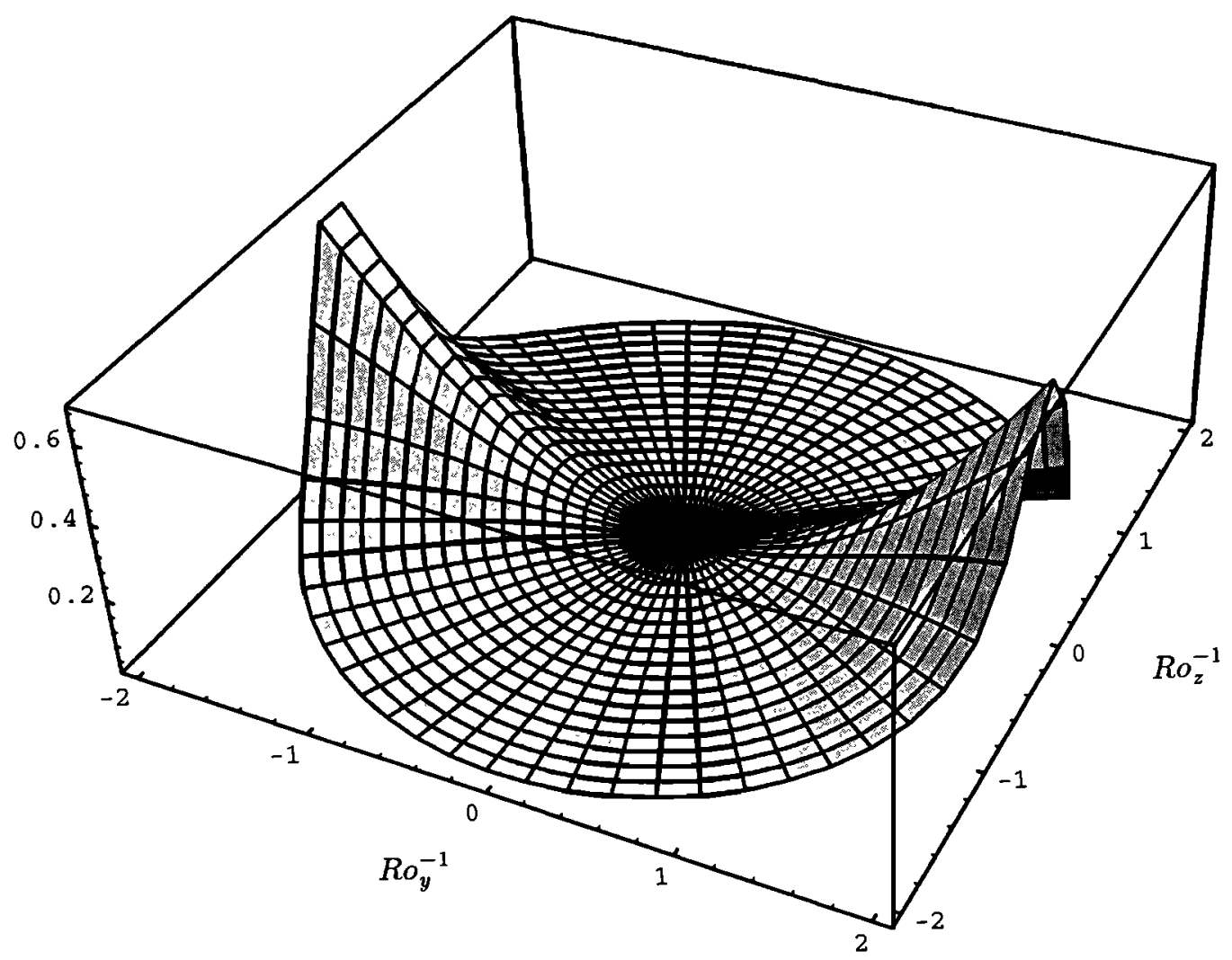

Figure 1. Limiting value for $G_{H}$ for purely convective turbulence.

\section{Dependence of Exchange Coefficients and Turbulence Energy Components on the Model Parameters}

Figures 3 through $4 \mathrm{e}$ describe variation of turbulence characteristics in unstably stratified flows. The results for neutral flows generally exhibit behavior similar to that in Figures 3 through $4 \mathrm{e}$, although the range of variation may be reduced; thus these results are not shown here. A general comment should be made. Although $R u, R v, G_{H}, R o_{y}^{-1}$, and $R o_{z}^{-1}$ appear as free parameters in (1)-(4), they are not so in the full model that calculates mean profiles using eddy viscosity and diffusivity. Therefore Figures 3-4e should be viewed as an illustration of the possible range of variation of the turbulence variables rather than as actual configurations. For example, in Figure 3 for $R o_{y}^{-1} \geq 1$ and $R o_{z}^{-1}=0$ one finds that $\overline{u^{2}} / q^{2}+\overline{v^{2}} / q^{2}+\overline{w^{2}} / q^{2} \approx 0.65<1$. However, it is unlikely that such a situation can occur in simulations with prognostic $R u, R v$, and $G_{H}$, when realizability conditions are enforced.

In Figure 3 the dependence of the components of the turbulence energy and the eddy diffusivity on $R_{z}^{-1}$ and $R o_{y}^{-1}$ is shown for purely convective turbulence $(R u=R v=0)$. One can see that the dependence on $R o_{z}^{-1}$ disappears for $R o_{y}^{-1}=0$, in agreement with observations made earlier. Consistently with the model's equations, $\overline{w^{2}} / q^{2}$ and $S_{\theta \theta}$ vary proportionally; the dependence on $R o_{y}^{-1}$ is significant for all parameters, par- ticularly for $\overline{w^{2}} / q^{2}$ and $S_{\theta \theta}$. The behavior of $\overline{u^{2}} / q^{2}$ and $\overline{v^{2}} / q^{2}$ is complex, but the range of their variation is small. Note that Figure 3 is symmetrical with respect to the $R o_{y}^{-1}=0$ axis.

Figures 4a through $4 \mathrm{e}$ show model results for shear convective turbulence, where it was set $G_{H}=0.02$ and $\left(R u^{-2}+R v^{-2}\right)^{1 / 2}=0.4$. These results depend not only on the magnitude of the velocity vector, but also on its direction; there are significant differences for negative and positive values of $R u$ and $R v$. Figures $4 \mathrm{a}$ and $4 \mathrm{~b}$ indicate that, similarly to the tendencies found in Galperin and Kantha [1989] in spanwise rotating flows, the mean shear acts to redistribute turbulence energy between $\overline{u^{2}}$ and $\overline{w^{2}}$, such that increase/decrease in the former is accompanied by decrease/increase in the latter. However, the presence of nonzero $f$ makes the picture more subtle because, contrary to the case considered by Galperin and Kantha [1989], $\overline{v^{2}}$ is now also a function of $R o_{y}^{-1}$ and $R o_{z}^{-1}$. Consistent with the analysis in section 3.1, the mixing coefficients shown in Figures $4 \mathrm{c}$ and $4 \mathrm{~d}$ may vary by almost an order of magnitude, even for small values of $\left|R o_{y}^{-1}\right|$ and $\left|R o_{z}^{-1}\right|$. Generally, large $\left|R o_{y}^{-1}\right|$ and $\left|R o_{z}^{-1}\right|$ tend to reduce $S_{u u}$ (Figure 4c), consistent with the results of Galperin and Kantha [1989]. However, this tendency to stabilization may now be offset by the increase in $S_{v v}$, which has a destabilizing nature. Also, the stabilizing influence of the Coriolis terms often more than compensates the destabilization due to the thermal forces. For moderately negative values of $R u, R v$, and $R o_{y}^{-1}$, both $S_{u u}$ and 

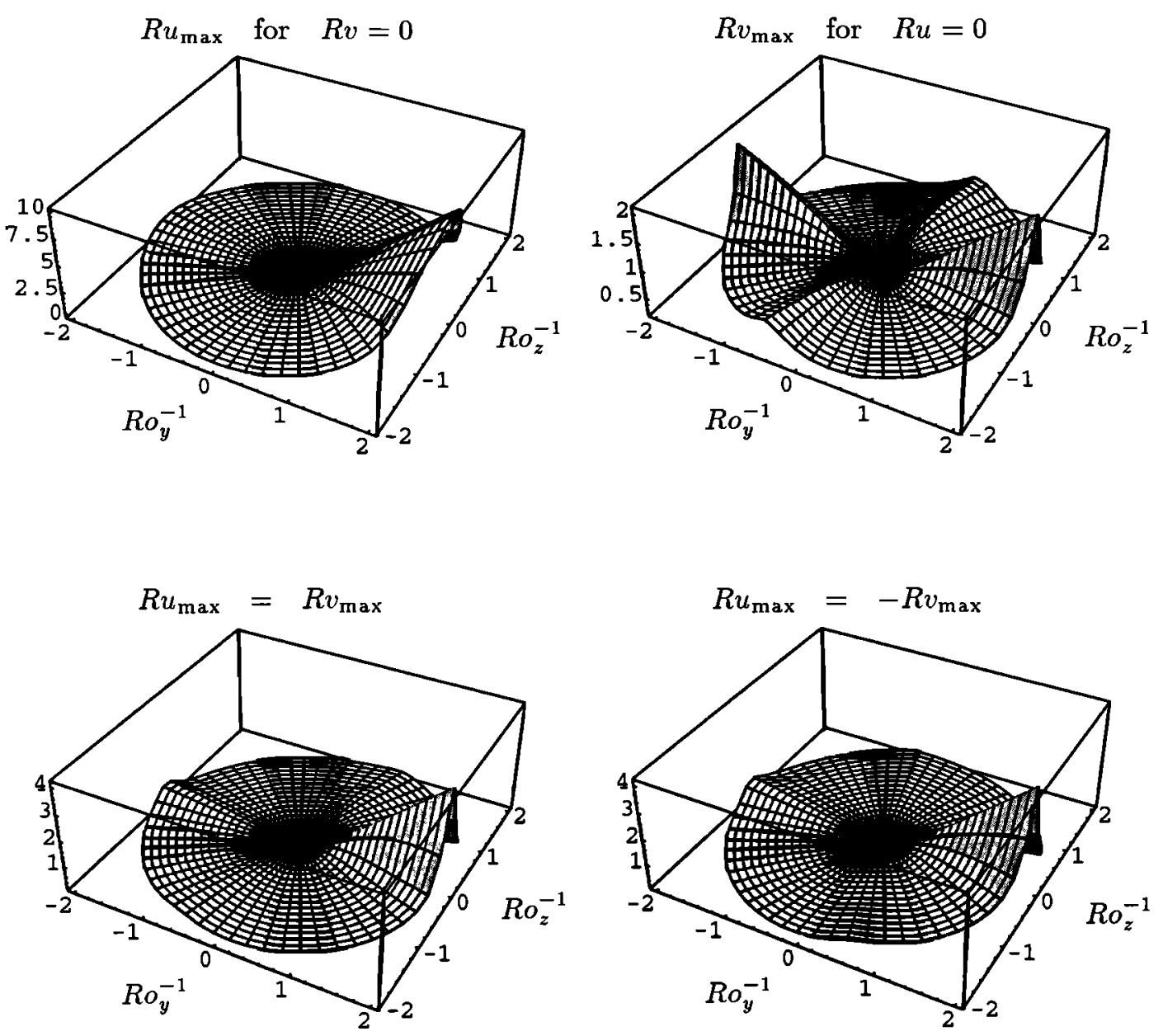

Figure 2. Limiting values for $R u$ and $R v$ for convective turbulence; $G_{H}\left(R u^{-2}+R v^{-2}\right)^{-1 / 2}=$ 0.05 .

$S_{v v}$ increase, and the flow is rather destabilized. The decrease in $S_{u u}$ for larger negative values of $R u$ and $R v$ can be attributed to restabilization; a tendency somewhat similar to that discussed in Galperin and Kantha [1989].

In all cases the off-diagonal components of the eddy viscosity-eddy diffusivity tensor that were identical zero for $R o_{z}^{-1}=R o_{y}^{-1}=0$ significantly increase with increasing absolute values of the inverse Rossby numbers, as seen in Figure 4d. They become comparable with the diagonal terms (Figure 4c), particularly for negative $R u$ and $R v$. Sometimes, the $S_{u r} \partial U / \partial z$ term in (9) may be smaller than the other terms in the $\overline{u w}$ equation, which indicates that in actual simulations it may be necessary to simultaneously invert the entire matrix rather than estimate the off-diagonal terms from the last time step, a procedure that may lead to numerical instability.

\section{Comparison With Other Experimental Results}

A partial test for the model is the experiments of Fernando et al. [1991], in which the characteristics of convective turbulence in the presence of axial rotation
$\left(R o_{y}^{-1}=0, R o_{z}^{-1} \neq 0\right)$ were studied. For such flows, model equations simplify significantly.

In the experiments of Fernando et al. [1991] there are two regimes that admit self-similar solutions (1) the developing boundary layer, where the mixed layer entrains into the quiescent fluid, and (2) the fully mixed regime, where the mixed layer fills the entire domain. In both cases one can relate the turbulence energy to the buoyancy flux $Q$ by invoking the assumption of local equilibrium,

$$
\begin{aligned}
& Q=q^{3} / B_{1} l, \\
& Q \equiv-\beta g \overline{w \theta} .
\end{aligned}
$$

It was found that in almost all the experiments the turbulence macroscale had attained the values at which it became limited by centrifugal forces and could be calculated from the equation

$$
\Omega l=q .
$$

Equation (22) is consistent with (13c). Substituting (22) with (20), one obtains an expression for turbulence energy in the vicinity of a wall $q_{0}$, where the buoyancy flux is $Q_{0}$ :

$$
q_{0}\left(Q_{0} / \Omega\right)^{-1 / 2}=B_{1}^{1 / 2} .
$$



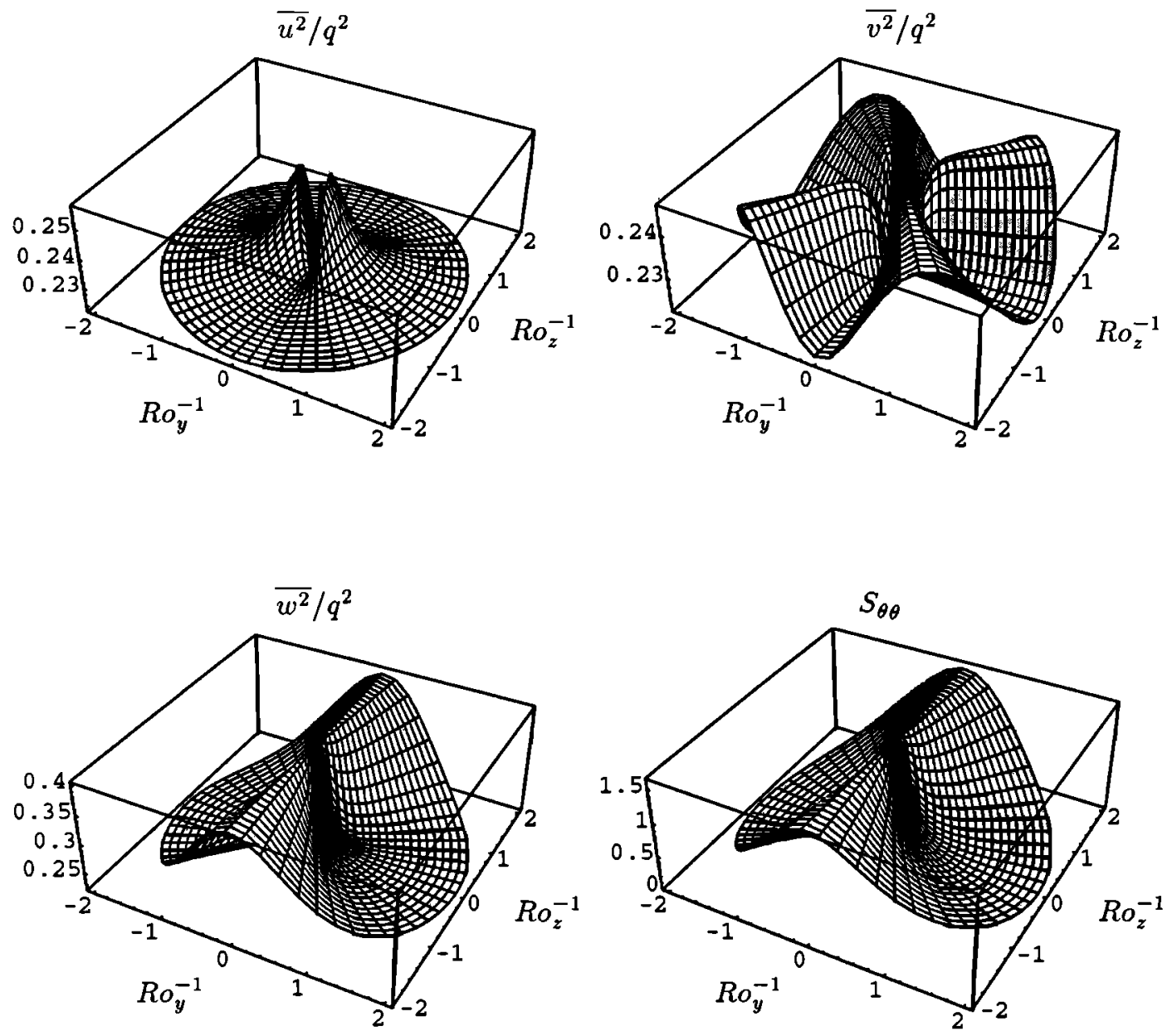

Figure 3. Dependence of $\overline{u^{2}} / q^{2}, \overline{v^{2}} / q^{2}, \overline{w^{2}} / q^{2}$, and $S_{\theta \theta}$ on $R o_{y}^{-1}$ and $R o_{z}^{-1}$; convective turbulence $G_{H}=0.02$.

The value of the constant on the right-hand side of (23) is 4.07 , in fair agreement with the value of $2.5 \pm 0.3$ found by Fernando et al. [1991]. The equation for the buoyancy flux in terms of the buoyancy parameter $b \equiv$ $\beta g \Theta$ is

$$
Q=C q l \frac{\partial b}{\partial z}
$$

where the constant $C$ can be derived from the present model. Assuming that $G_{H}$ has reached its limiting value of 0.0233 and that the dependence of $S_{\theta \theta}$ on $G_{H}$ has ceased, one finds $C \cong 2.52$. Then, using (20), (21), and (24), it can be shown that

$$
\frac{\partial b}{\partial z}=-\frac{\Omega^{2}}{C B_{1}} .
$$

Thus the buoyancy gradient is constant and proportional to $\Omega^{2}$, the constant of proportionality being equal to 0.025 , as compared with a value of 0.02 , which is consistent with the measurements of Fernando et al. [1991] for the fully mixed regime. The buoyancy (temperature) profile is linear, varying from $\Omega^{2} h_{r} /\left(C B_{1}\right)$ at $z=0$ to 0 at the edge of the mixed layer, where $z=h_{r}$. The buoyancy balance equation becomes

$$
\frac{d}{d t}\left(\frac{b h_{r}}{2}\right)=Q_{0}
$$

giving

or

$$
h_{r}^{2}=\frac{2 C B_{1} Q_{0} t}{\Omega^{2}},
$$

$$
h_{r}\left(\Omega^{-3} Q_{0}\right)^{-1 / 2}=\left(2 C B_{1} \Omega t\right)^{1 / 2} .
$$

The variation of $h_{T}$ is compared with the data of Fernando et al. [1991] in Figure 5. In the same figure the model-predicted curve for no rotation is shown to be in excellent agreement with $h_{r}=0.3\left(Q_{0} t\right)^{3 / 2}$, which correlates with Fernando et al.'s no-rotation data (note that in plotting this correlation we multiplied both its sides by $\left.\left(\Omega^{-3} Q_{0}\right)^{-1 / 2}\right)$. Consistent with the experiments, the present results show that rotation decreases the rate of entrainment of the mixed layer by an order of magnitude. The observed development of the mixed layer is relatively well predicted for most experiments. In some cases, though, for low values of heat flux and high angular velocity, the Fernando et al. data are rather underpredicted. In these cases, Fernando et al. state that the viscous effects are rather important and cannot be neglected. 
$$
\overline{u^{2}} / q^{2}
$$
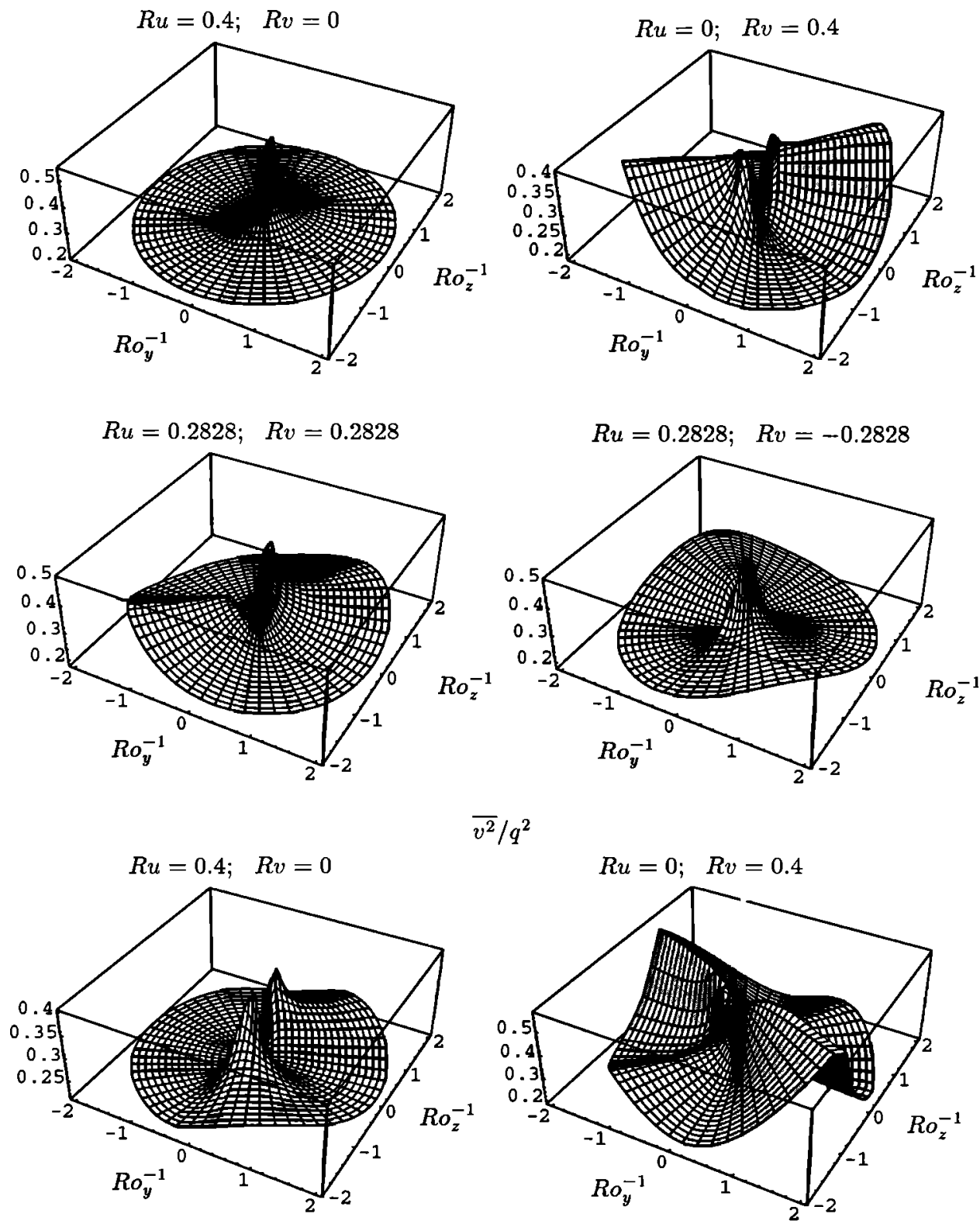

$\overline{v^{2}} / q^{2}$
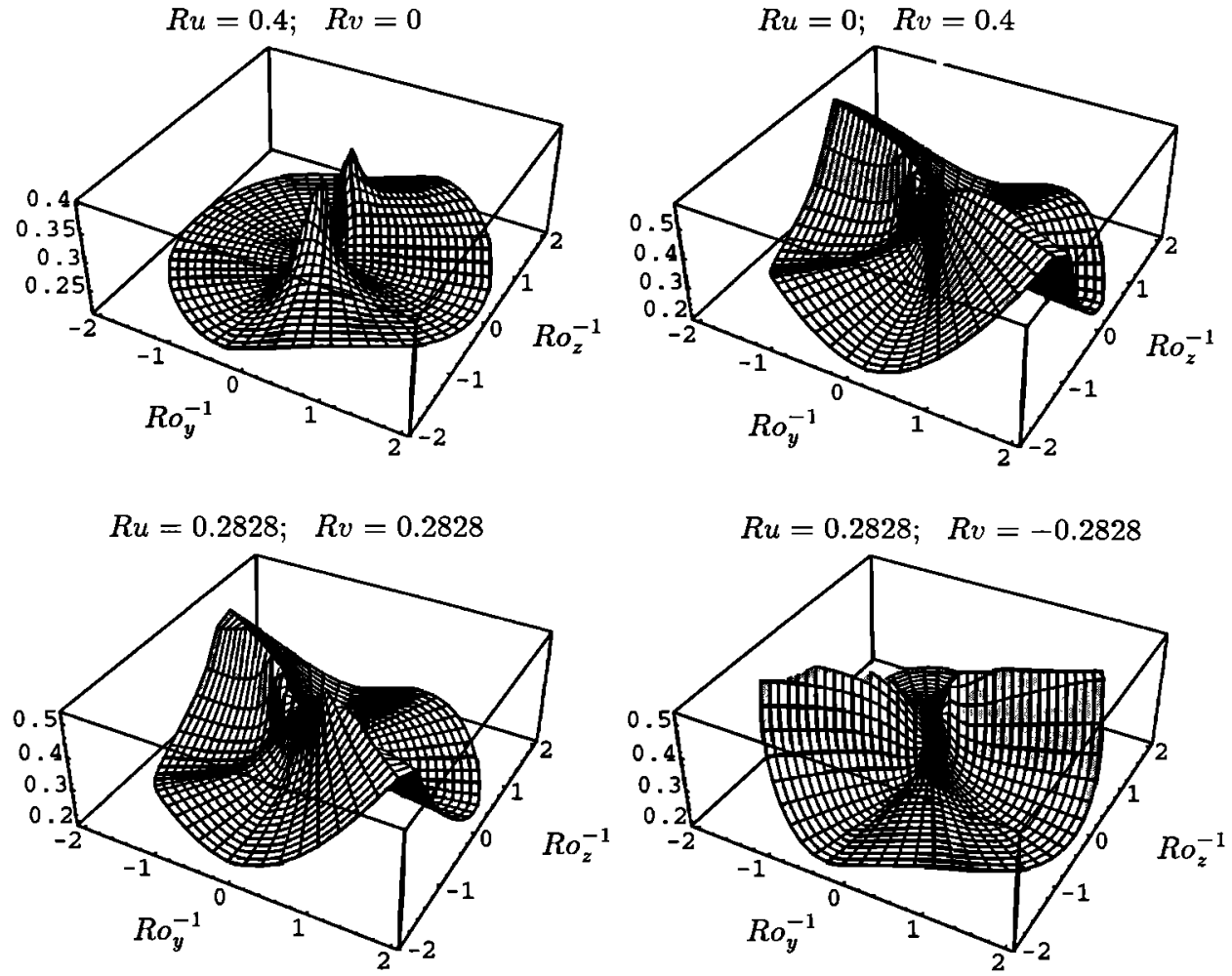

Figure 4a. Dependence of $\overline{u^{2}} / q^{2}$ and $\overline{v^{2}} / q^{2}$ on $R o_{y}^{-1}$ and $R o_{z}^{-1} ; G_{H}=0.02$. 


$$
\overline{w^{2}} / q^{2}
$$
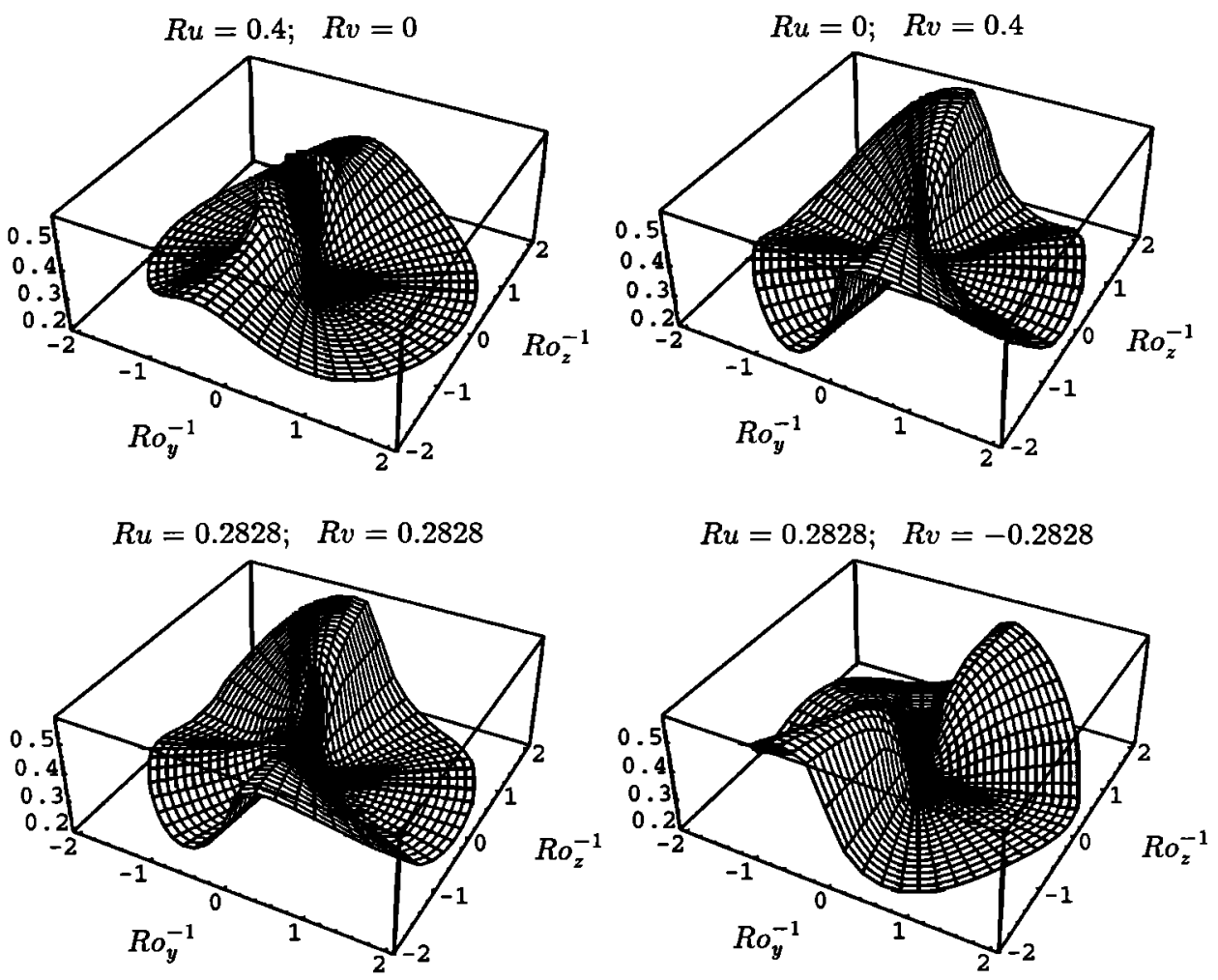

$S_{\theta \theta}$
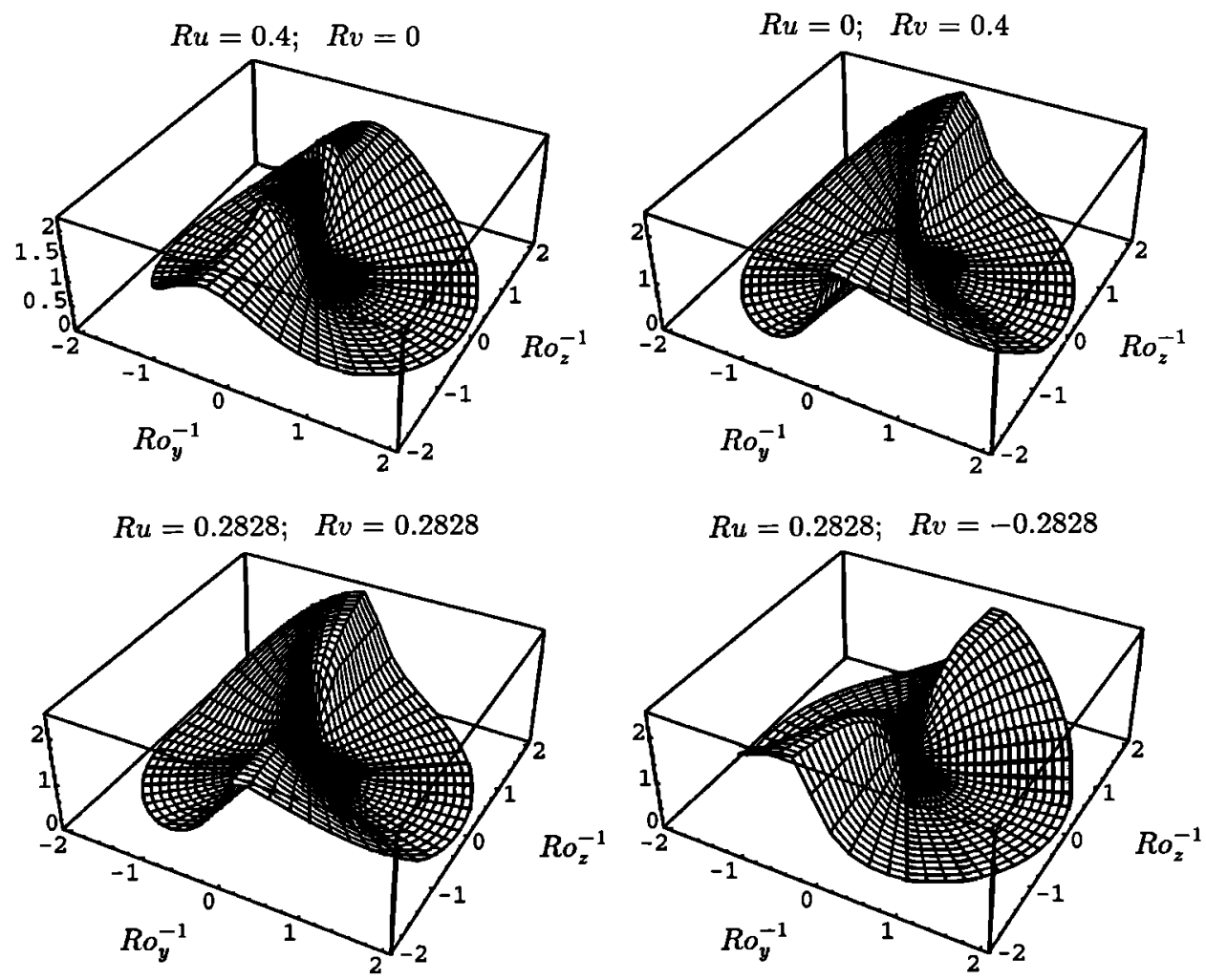

Figure 4b. Dependence of $\overline{w^{2}} / q^{2}$ and $S_{\theta \theta}$ on $R o_{y}^{-1}$ and $R o_{z}^{-1} ; G_{H}=0.02$. 


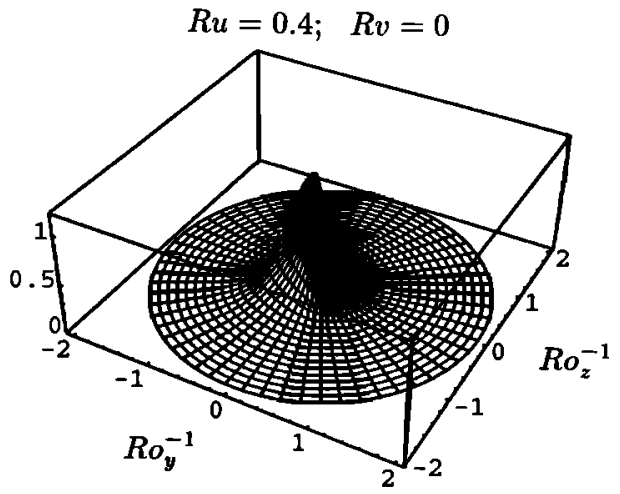

$S_{u u}$

$R u=0.2828 ; R v=0.2828$
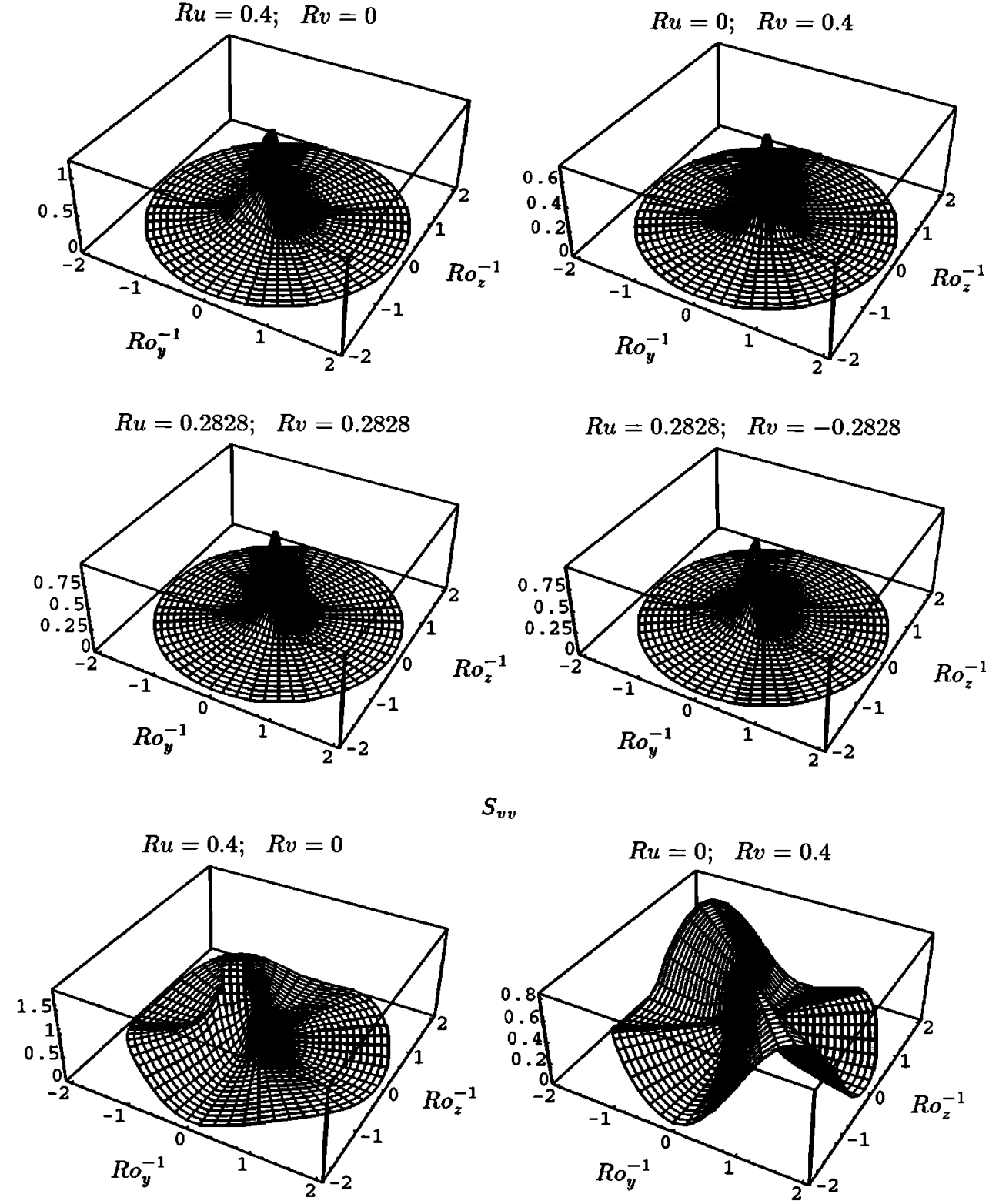

$S_{v v}$
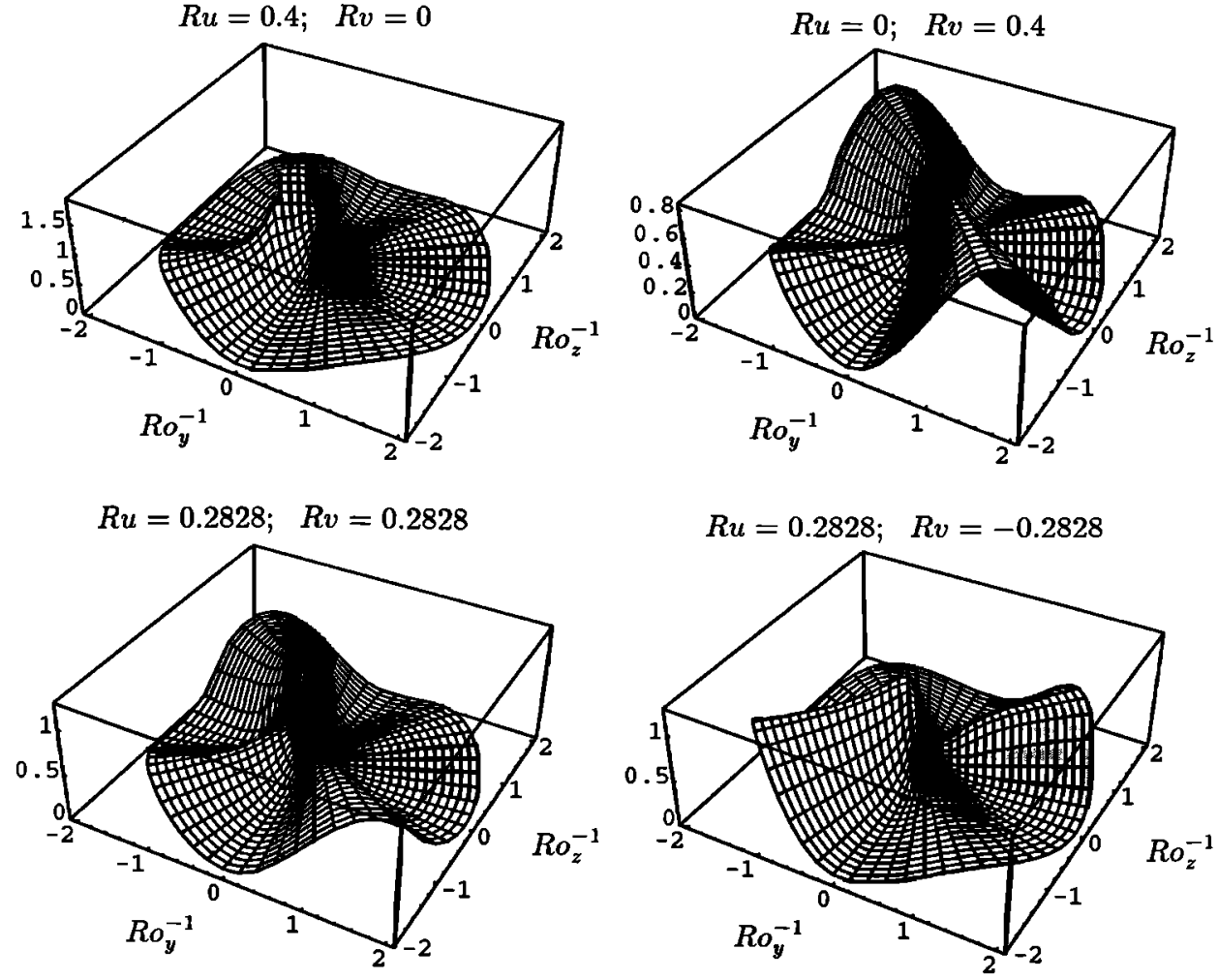

Figure 4c. Dependence of the diagonal mixing coefficients $S_{u u}$ and $S_{v v}$ on $R o_{y}^{-1}$ and $R o_{z}^{-1}$; $G_{H}=0.02$. 
$S_{u v}$
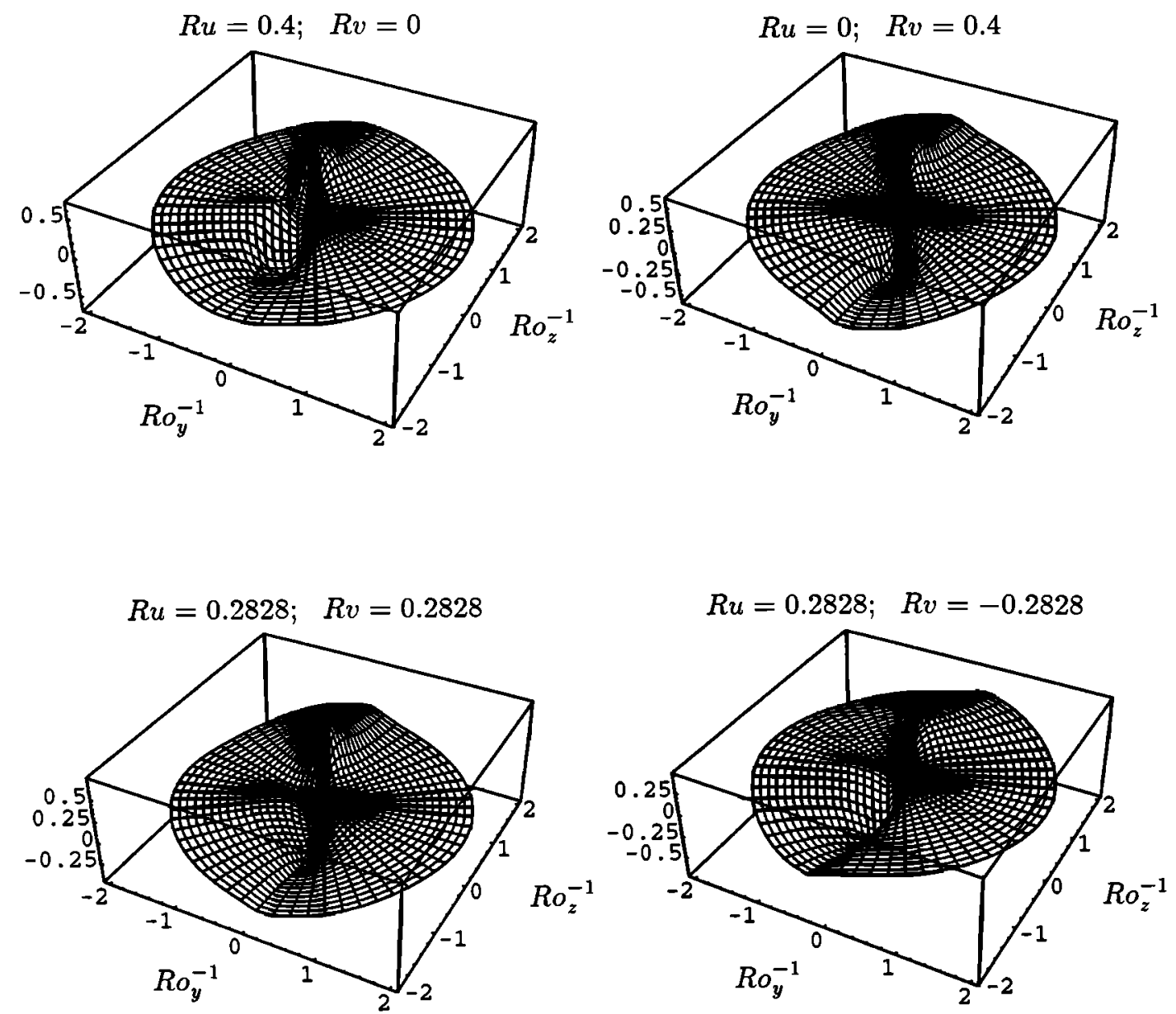

Figure 4d. Dependence of the off-diagonal mixing coefficient $S_{u v}\left(\simeq S_{v u}\right)$ on $R_{y}^{-1}$ and $R o_{z}^{-1}$; $G_{H}=0.02$.

The buoyancy variance can also be calculated assuming local equilibrium between its production and dissipation

$$
\overline{b^{\prime 2}}=-B_{2} \frac{l}{q} Q \frac{\partial b}{\partial z}=B_{2} \frac{Q \Omega}{C B_{1}},
$$

or for near-wall values,

$$
\left(\overline{b_{0}{ }^{\prime 2}}\right)^{1 / 2}=\left(\frac{B_{2}}{B_{1} C}\right)^{1 / 2}\left(\Omega Q_{0}\right)^{1 / 2} .
$$

The coefficient in front of $\left(\Omega Q_{0}\right)^{1 / 2}$ in (30) is equal to 0.5 , in good agreement with the measurements of Fernando et al. [1991] for the middle part of the boundary layer, from which a value of $0.3 \pm 0.1 \mathrm{can}$ be inferred. The measurements of Boubnov and Golitsyn [1990] give a close value of 0.648 .

It can be seen that the agreement with the experimental data of Fernando et al. [1991] is good for mean flow parameters and buoyancy variance and fair for turbulent velocity. The general form of the equations is the same, and the scaling laws based on the angular velocity are consistent with the results of Fernando et al.

It is important to note that, contrary to the case of pure convection, studies of turbulence in rotating fluids conducted in experimental apparatuses of finite dimensions are invariably accompanied by secondary circulation, which affects the growth rate of the mixed layer (see, for instance, Boubnov and Golitsyn [1990] and Hassid et al. [1991]). Therefore, to improve the agreement with the data of Fernando et al. [1991], it may be necessary to fully account for the effects of the secondary circulation, as well as for molecular effects; a task beyond the scope of this effort. However, the experimental results of Fernando et al. do support the assumed form of the length scale limitation in the presence of background rotation. Without such a limitation it would be impossible to obtain any kind of agreement between the scaling laws derived from the present model and the experimental results.

\section{Discussion and Conclusions}

The analysis of the direct effects of Coriolis terms on the Reynolds stress and heat flux equations carried out in paper 1 for stably stratified flows, has been extended here to neutral and unstably stratified flows. The assertion made in paper 1 that in the absence of limiting 
$S_{u \theta}$
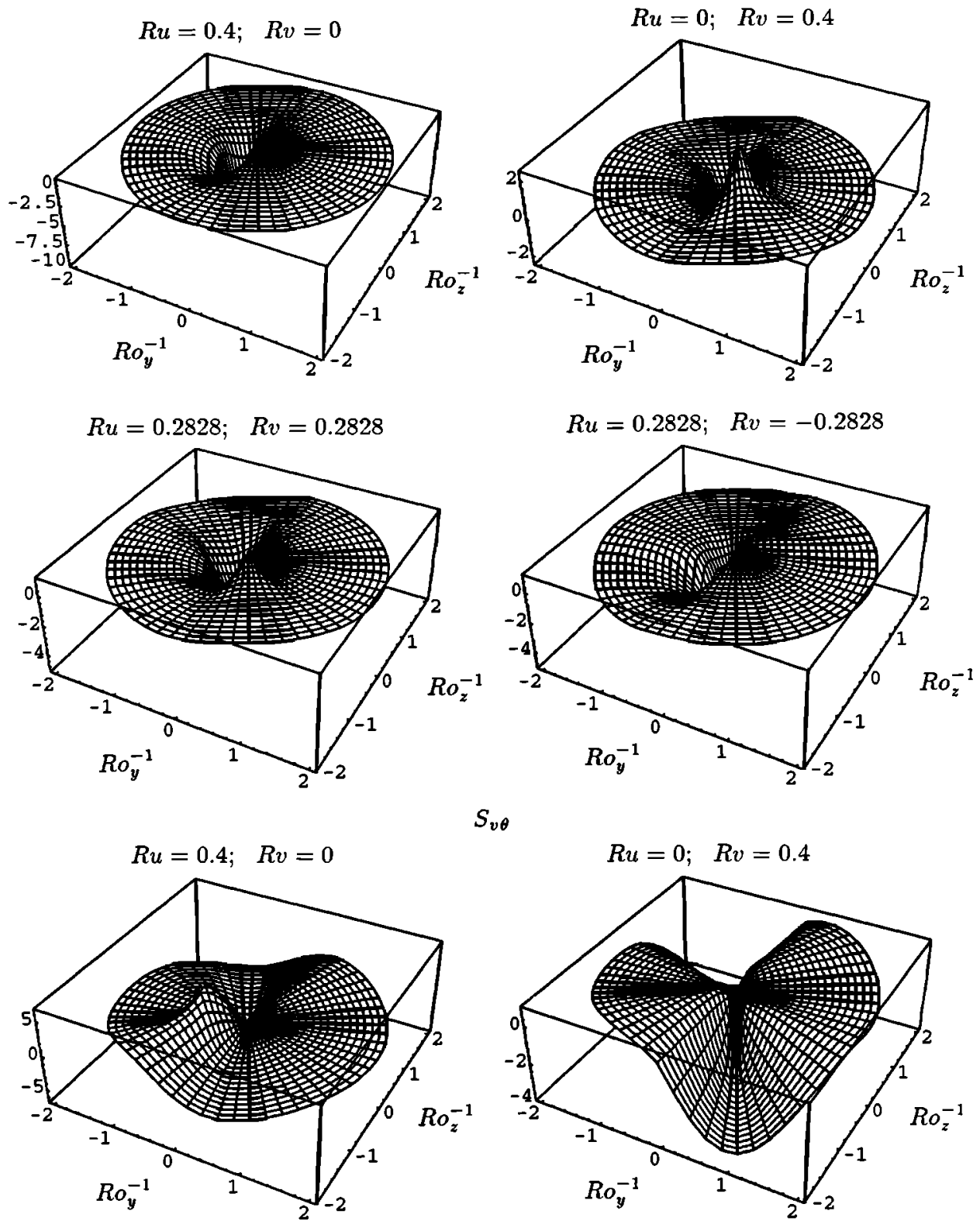

$S_{v \theta}$
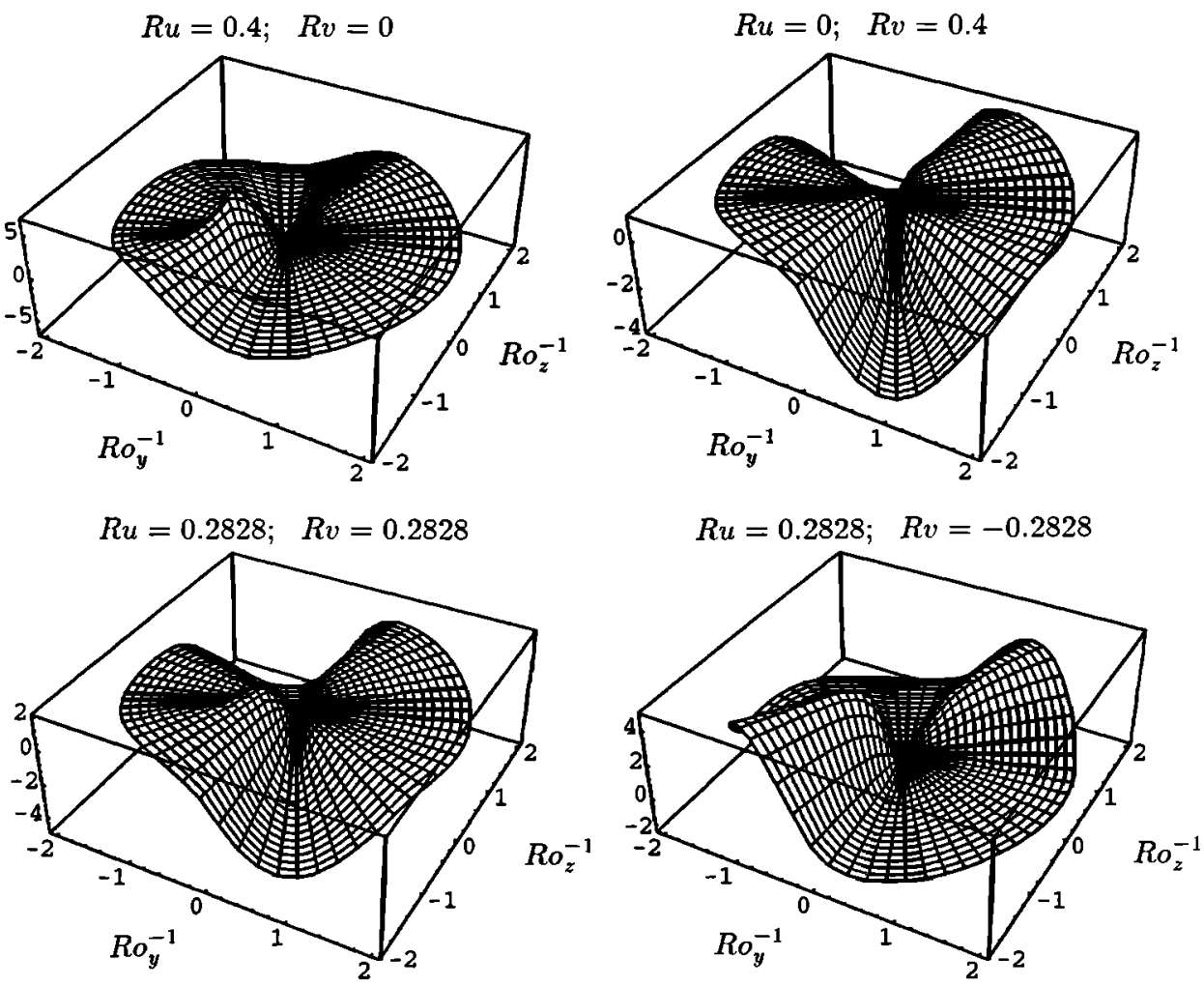

Figure 4e. Dependence of $S_{u \theta}$ and $S_{v \theta}$ on $R o_{y}^{-1}$ and $R o_{z}^{-1} ; G_{H}=0.02$. 


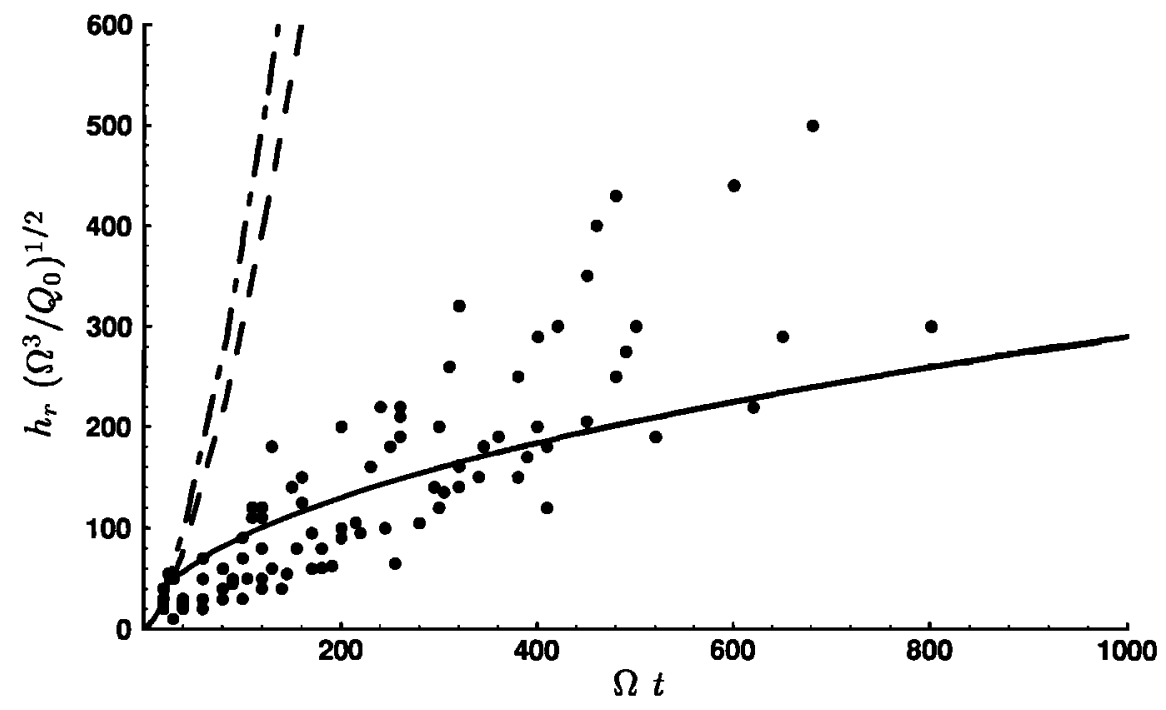

Figure 5. Development of the convective mixed layer with rotation. Solid line is model predictions (equation (28)); circles are data of Fernando et al. [1991] for $Q_{0}=7.1^{-7} \mathrm{~m}^{2} \mathrm{~s}^{-3}$ and $2.2510^{-6} \mathrm{~m}^{2} \mathrm{~s}^{-3}$. For comparison, development of the convective mixed layer without rotation is also shown; dashed line is the correlation developed by Fernando et al., dotted-dashed line is the prediction of the present model.

stable stratification, rotational contribution in turbulence correlation equations may be equal to or even exceed contributions from other terms, has been confirmed. Furthermore, it was found that there are ranges of the model's parameters in which the system becomes physically unrealizable. To alleviate such problems, a set of rules or realizability conditions has been established that prevents the system from developing singular or nonphysical behavior. These conditions result from generalization of a similar approach described in paper 2 but are much more complex algebraically, reflecting the complexity of the present model.

An important constraint developed in the present study is the length scale limitation due to rotation. Although its physical mechanism differs from that of stable stratification, it acts in a similar way, limiting the maximum size the turbulence macroscale can attain in a rotating coordinate system. It is important to note that neither the length scale limitation due to stable stratification nor that due to rotation can be derived from the present model or other conventional Reynolds stress closure models; both should be imposed on the model as external constraints. The roots of this deficiency stem from the inability of the Reynolds average closure models to describe flows that undergo transition from 3-D to 2-D turbulence under the action of external factors or extra strains. For stably stratified flows, such a transition takes place at scales comparable with the Dougherty-Ozmidov scale, $L_{O}=\left(\epsilon / N^{3}\right)^{1 / 2}$; $\epsilon$ being the rate of the viscous dissipation [Dougherty, 1961; Ozmidov, 1965; Stillinger et al., 1983; Itsweire et al., 1993]. For rotating flows the relevant scale is $L_{\Omega}=\left(\epsilon / \Omega^{3}\right)^{1 / 2}[$ Kamenkovich et al., 1986]. The length scale limitations employed in the present model for both types of flow are closely related to $L_{O}$ (see paper 1) and $L_{\Omega}$.
The results of sections 3.1 and 4 clearly demonstrate that the Coriolis effect has an appreciable influence on the turbulence structure. The mixing coefficients assume a tensorial form with significant off-diagonal terms. In paper 1 , it was shown in a very general way that in the range of geophysically relevant parameters the stable stratification effectively limits the total contribution of Coriolis terms to turbulence correlations, bounding it to within $10 \%$ of the other terms. In the present study it was shown that in neutral and convective flows, rotation can change the rates of momentum and heat transfer by an order of magnitude even when $R o_{y}^{-1}$ and $R o_{z}^{-1}$ are relatively small and thus the Coriolis effects by no means can be neglected. Moreover, given that the destabilizing influence of stratification is sometimes more than counterbalanced by the stabilizing influence of rotation, excluding these effects may result in a seriously faulty image of the turbulent exchange processes.

To implement the expressions developed in this paper in models of oceanic and/or atmospheric circulation, one will have to overcome difficulties related to the complexity of the mathematical expressions for the exchange coefficients; the necessity of using matrix equations and the need to solve a transcendental equation to find the constraints on the dimensionless parameters of the model. Although these difficulties are appreciated, the use of the simplified expressions may not be an appropriate solution in the general case. In particular, although the expressions obtained for vertical and poleward rotation separately are significantly simpler than the general case, the interaction of the two components of rotation is too complex to be described by simplified models. This is particularly true for the poleward component, which can have a destabilizing influence for some values of the velocity gradient and a stabilizing in- 
Huence for others, as explained by Galperin and Kantha [1989].

The present study shows that if Reynolds stress closure models are to be used to parameterize neutral and, particularly, convective mixed layers in the oceanic environment, then they should include Coriolis terms in all equations describing second-order turbulence correlations and thus vertical mixing coefficients. Given this additional complexity and the fact known mostly from the meteorological research that eddy viscositydiffusivity formulation with a single-turbulence macroscale cannot reproduce certain features of convective boundary layers (see, for example, Galperin and Hassid [1986], and references therein), it is useful to reassess the utility of Reynolds average models for describing vertical mixing in OGCMs. Let us consider some alternatives. In principle, one could think of direct numerical simulation of convective turbulence, when a system of governing equations is solved for all scales, including the viscous dissipation. Obviously, such an approach is unfeasible for realistic spatial and temporal scales. Some success in modeling of convective flows has been achieved using another alternative, large-eddy simulation (LES) (see, for instance, Mason [1989], Schmidt and Schumann [1989], or Galperin and Orszag [1993] for a review). However, this method requires parameterization of unresolved or subgrid scales of motion. To make the method computationally efficient, the subgrid scales should extend to relatively large scales, at which, as suggested by the present study, the effects of unstable stratification and rotation are significant, leading to the same kind of difficulties as those described in section 2. Although LES is capable of simulating flows with complex physics, proper subgrid scale parameterization that includes the effects of unstable stratification and rotation is still a subject of ongoing research, and thus prohibitively high resolution is required in such simulations. Finally, as yet another alternative, Jones and Marshall [1993] have recently performed high-resolution simulations (about $240 \mathrm{~m} \times 240 \mathrm{~m} \times 100 \mathrm{~m}$ ) of nonhydrostatic deep convection. To parameterize turbulent mixing, they used eddy viscosity-eddy diffusivity formulation with constant horizontal and vertical viscosity coefficients (they assumed that the corresponding viscosities and diffusivities are equal). Results reported by Jones and Marshall resemble some features of convective flows, and some experimental scaling laws have also been reproduced. However, these simulations are similar to LES and have similar shortcomings. In particular, even with the relatively high resolution used in these simulations, there is still considerable amount of subgrid-scale energy residing on unresolved scales, and using constant eddy viscosity and diffusivity does not seem to be well justified.

Reynolds average modeling can be considered as a limiting case of LES in which all scales have been eliminated, and the resulting description mostly pertains to the mean flow and turbulence correlations. This method is more efficient than LES because it does not require high resolution and allows, in some cases, for systematic simplifications [Mellor and Yamada, 1974].
Reynolds average models account for the small-scale, 3-D, near-isotropic turbulence responsible for the vertical, quasi $1-D$ mixing in systems with large aspect ratio and, thus they can be and are being used in atmospheric and oceanic GCMs (for example, see Rosati and Miyakoda, [1988]); the horizontal, quasi 2-D mixing processes in these models are described by different parameterization schemes.

As a result of this study, it now appears necessary to retain Coriolis terms in equations for turbulence correlations and make necessary modifications in resulting eddy viscosity-diffusivity formulations in order to properly simulate atmospheric and oceanic boundary layers with background rotation and neutral and unstable stratification and, particularly, the deep convection episodes. Clearly, further research is necessary, particularly with respect to the assessment of the rotational effects on large-scale, long-term circulation and simplification of the turbulence closure scheme that would improve its computational efficiency.

Acknowledgments. The authors are grateful to anonymous reviewers whose comments helped to improve the manuscript. This research has been partially supported by ONR grant N00014-92-J-1363, NSF grant OCE 9010851, and the Fund for the Promotion of Research at the TechnionIsrael Institute of Technology.

\section{References}

André, J.C., G. De Moor, P. Lacarrere, G. Thery, and R. du Vachat, Modelling the 24 hour evolution of the mean and turbulent structures of the planetary boundary layer, J. Atmos. Sci., 95, 1861-1883, 1978.

Blumberg, A.F., and G.L. Mellor, Diagnostic and prognostic numerical circulation studies of the South Atlantic Bight, J. Geophys. Res., 88, 4579-4592, 1983.

Boubnov, B.M., and G.S. Golitsyn, Temperature and velocity field regimes of convective motions in a rotating plane fluid layer, J. Fluid Mech., 219, 215-239, 1990.

Cambon, C., and L. Jacquin, Spectral approach to nonisotropic turbulence subjected to rotation, J. Fluid Mech., 202, 295-317, 1989.

Dougherty, J.P., The anisotropy of turbulence of the meteor level, J. Atmos. Terr. Phys., 21, 210-213, 1961.

Fernando, H.J.S., R.R. Chen, and D.L. Boyer, Effects of rotation on convective turbulence, J. Fluid Mech., 228, 513-547, 1991.

Galperin, B., and S. Hassid, A modified turbulent energy model for geophysical flows: Influence of the ground proximity. Boundary-Layer Meteorol., 35, 155-165, 1986.

Galperin, B., and L.H. Kantha, A turbulence model for rotating flows. AIA A J., 27, 750-757, 1989.

Galperin, B., L.H. Kantha, S. Hassid, and A. Rosati, A quasi-equilibrium turbulent energy model for geophysical flows. J. Atmos. Sci., 45, 55-62, 1988.

Galperin, B., and G.L. Mellor, The effects of streamline curvature and spanwise rotation on near-surface, turbulent boundary layers, ZAMP, 42, 565-583, 1991.

Galperin, B., and S.A. Orszag (Eds.), Large Eddy Simulation of Complex Engineering and Geophysical Flows, Cambridge University Press, New York, 1993.

Galperin, B., A. Rosati, L.H. Kantha, and G.L. Mellor, Modeling rotating stratified turbulent flows with application to oceanic mixed layers, J. Phys. Oceanogr., 19, 901-916, 1989. 
Hakkinen, S., G.L. Mellor, and L.H. Kantha, Modeling deep convection in the Greenland Sea, J. Geophys. Res., 97, 5389-5408, 1992.

Hassid, S., E. Kit, and E. Mazor, Turbulent flow generated in an annulus by a rotating screen, Acta Mech., 86, 167177, 1991.

Itsweire, E.C., J.R. Koseff, D.A. Briggs, and J.H. Ferziger, Turbulence in stratified shear flows: Implications for interpreting shear-induced mixing in the ocean, J. Phys. Oceanogr., 23, 1508-1522, 1993.

Jacquin, L., O. Leuchter, C. Cambon, and J. Mathieu, Homogeneous turbulence in the presence of rotation, J. Fluid Mech., 220, 1-52, 1990.

Jones, H., and J. Marshall, Convection with rotation in a neutral ocean: A study of open-ocean deep convection, $J$. Phys. Oceanogr., 23, 1009-1039, 1993.

Kamenkovich, V.M., M.N. Koshljakov, and A.S. Monin (Eds.), Synoptic Eddies in the Ocean, D. Riedel, Norwell, Mass., 1986.

Kantha, L.H., A. Rosati, and B. Galperin, Effect of rotation on vertical mixing and associated turbulence in stratified fluids, J. Geophys. Res., 94, 4843-4854, 1989.

Mason, P.J., Large eddy simulation of the convective atmospheric boundary layer, J. Atmos. Sci., 46, 1492-1516, 1989

MEDOC Group, Observations of formation of deep water in the Mediterranean Sea, Nature, 227, 1037-1040, 1970.

Mellor, G.L., Analytic prediction of the properties of stratified planetary surface layers, J. Atmos. Sci., 30, 1061$1069,1973$.

Mellor, G.L., An equation of state for numerical models of oceans and estuaries. J. Atmos. Oceanic Technol., 8, 609-611, 1991.

Mellor, G.L., and H.J. Herring, A survey of the mean tur- bulent field closure models, AIAA J., 11, 590-599, 1972.

Mellor, G.L., and T. Yamada, A hierarchy of turbulence closure models for planetary boundary layers, J. Atmos. Sci., 31, 1791-1806, 1974.

Mellor, G.L., and T. Yamada, Development of a turbulence closure model for geophysical fluid problems, Rev. Geophys. Space Phys., 20, 851-875, 1982.

Ozmidov, R.V., On the turbulent exchange in a stably stratified ocean, Izv. Acad. Sci. USSR Atmos. Oceanic Phys. Engl. Transl., 8, 853-860, 1965.

Rosati, A., and K. Miyakoda, A general circulation model for upper ocean simulation, J. Phys. Oceanogr., 18, 1601$1626,1988$.

Schmidt, H., and U. Schumann, Coherent structure of the convective boundary layer deduced from large-eddy simulation, J. Fluid Mech., 200, 511-562, 1989.

Stillinger, D.C., K.N. Helland, and C.W. Van Atta, Experiments on the transition of homogeneous turbulence to internal waves in a stratified fluid, J. Fluid Mech., 191, 91-122, 1983.

Tritton, D.J., Stabilization and destabilization of turbulent shear flow in a rotating fluid, J. Fluid Mech., 241, 503523, 1992.

Boris Galperin, Department of Marine Science, University of South Florida, St. Petersburg, FL 33701.

Samuel Hassid, Environmental and Water Resources Engineering, Technion-Israel Institute of Technology, Haifa 32000 , Israel.

(Received January 8, 1993; revised July 29, 1993;

accepted October 21, 1993.) 